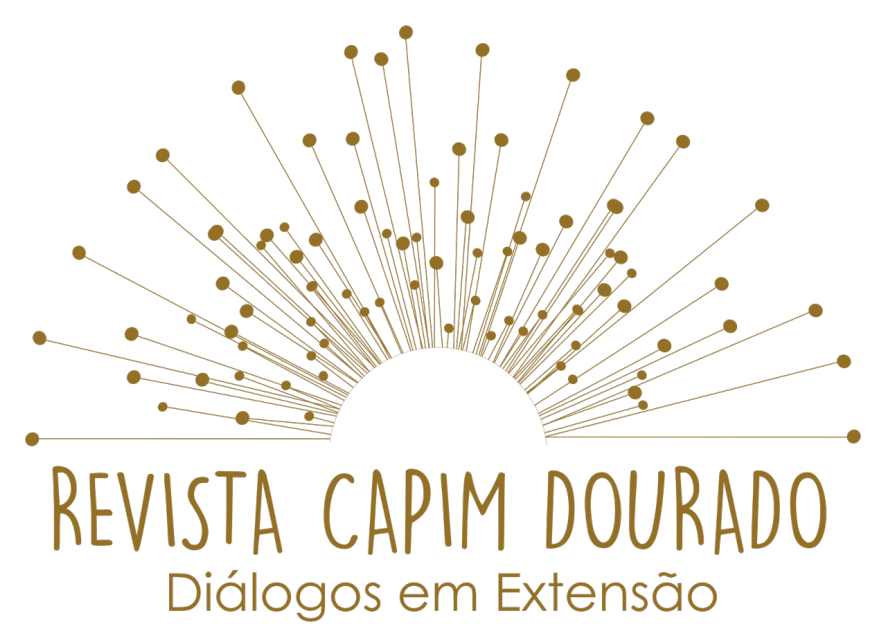

ISSN n² 2595-7341

Vol. 3, n. 1, Janeiro-Abril, 2020

DOI: http://dx.doi.org/10.20873/uft.2595-7341.2020v3n1p95

\title{
CARACTERÍSTICAS SOCIODEMOGRÁFICAS E ESTILO DE VIDA DE ADOLESCENTES DE TOCANTINÓPOLIS/TO
}

\author{
SOCIODEMOGRAPHIC CHARACTERISTICS AND LIFESTYLE OF \\ ADOLESCENTS FROM TOCANTINÓPOLIS / TO
}

CARACTERÍSTICAS SOCIODEMOGRÁFICAS Y ESTILO DE VIDA DE
ADOLESCENTES DESDE TOCANTINÓPOLIS / HASTA

\author{
Helizângela Morais Milhomem ${ }^{1}$ \\ Samuel Carvalho Da Silva ${ }^{2}$ \\ Idelvan Ferreira Da Silva ${ }^{3}$ \\ Sônia Cristina Alves Carvalho Leal ${ }^{4}$ \\ Joana Marcela Sales De Lucena ${ }^{5}$
}

\section{RESUMO}

Estudos têm demonstrado que o nível de atividade física está relacionado à manutenção de níveis saudáveis de aptidão física e, baixos níveis de aptidão física têm sido associados a o risco de desenvolver doenças cardiovasculares, de mortalidade por todas as causas, ao tabagismo e ao uso de bebidas alcoólicas. E tem como descrever a prevalência de atividade física, comportamentos

\footnotetext{
${ }^{1}$ Discente do curso de Licenciatura em Educação Física da Universidade Federal do Tocantins (UFT), Campus Tocantinópolis, Tocantinópolis, Tocantins, helynene2@hotmail.com.

${ }^{2}$ Discente do curso de Licenciatura em Educação Física da Universidade Federal do Tocantins (UFT), Campus Tocantinópolis, Tocantinópolis, Tocantins, samuel.carvalho@mail.uft.edu.br.

${ }^{3}$ Discente do curso de Licenciatura em Educação Física da Universidade Federal do Tocantins (UFT), Campus Tocantinópolis, Tocantinópolis, Tocantins, idelvan-sf-@hotmail.com.

${ }^{4}$ Discente do curso de Licenciatura em Educação Física da Universidade Federal do Tocantins (UFT), Campus Tocantinópolis, Tocantinópolis, Tocantins, soniacristina hyndya@hotmail.com.

${ }^{5}$ Professora do curso de Licenciatura em Educação Física da Universidade Federal do Tocantins (UFT), Campus Tocantinópolis, Tocantinópolis, Tocantins, joana.sales@mail.uft.edu.br.
} 


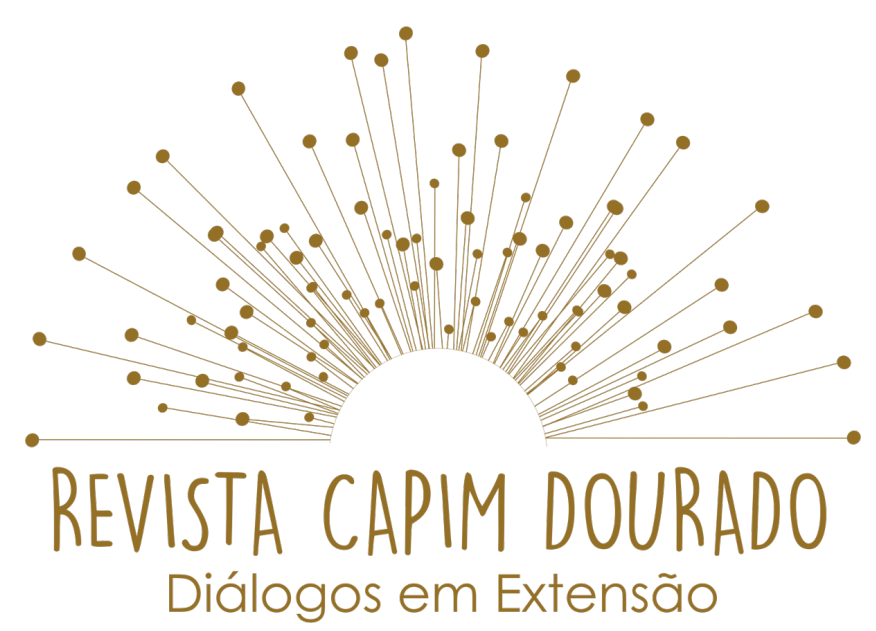

ISSN n² 2595-7341

Vol. 3, n. 1, Janeiro-Abril, 2020

DOI: http://dx.doi.org/10.20873/uft.2595-7341.2020v3n1p95

sedentários e suas possíveis associações com os fatores socioeconômicos e antropométricos de escolares da cidade de Tocantinópolis (TO). A coleta incluiu a aplicação de um questionário na forma de entrevista face a face, medida dos comportamentos sedentários considerou a duração (horas por dia) e a frequência (quantidade de dias na semana e no fim de semana) do uso, separadamente, de televisão, videogames e computador (no lazer e para atividades adolescentes) em dias de semana e fim de semana, considerando a semana anterior à coleta de dados atividade física foi mensurada por questionário validado Os adolescentes informaram a frequência (dias/semana) duração (minutos/dia) das atividades físicas praticadas na semana anterior à coleta de dados. Participaram do estudo 69 escolares com idade média de 15,4 anos ( $D P=0,2$ ), dos $6^{\circ}$ e $9^{\circ}$ ano do Ensino Fundamental e $1^{\circ}$ e $3^{\circ}$ ensino médio, dos quais $52,2 \%$ eram meninas. Os escolares realizavam em média $256,5 \mathrm{~min} . / \mathrm{dia}(\mathrm{DP}=29,9)$ de atividades físicas permaneciam 748,8 min./dia ( $D P=86,5$ ) assistindo televisão e 553,6 min./dia (DP $=75,9)$. Os resultados indicam que os adolescentes de Tocantinópolis/TO apresentam pouco tempo de atividade física e altos índices de comportamentos sedentários.

PALAVRAS-CHAVE: Atividade Física. Aptidão Física. Sedentarismo.

\section{ABSTRACT}

Studies have shown that the level of physical activity is related to the maintenance of healthy levels of physical fitness, and low levels of physical fitness have been associated with the risk of developing cardiovascular diseases, mortality from all causes, smoking and the use of beverages. alcoholic. And it can describe the prevalence of physical activity, sedentary behaviors and their possible associations with the socioeconomic and anthropometric factors of schoolchildren in the city of Tocantinópolis (TO). The collection included the application of a questionnaire in the form of a face-to-face interview, a measure of sedentary behaviors considering the duration (hours per day) and the 


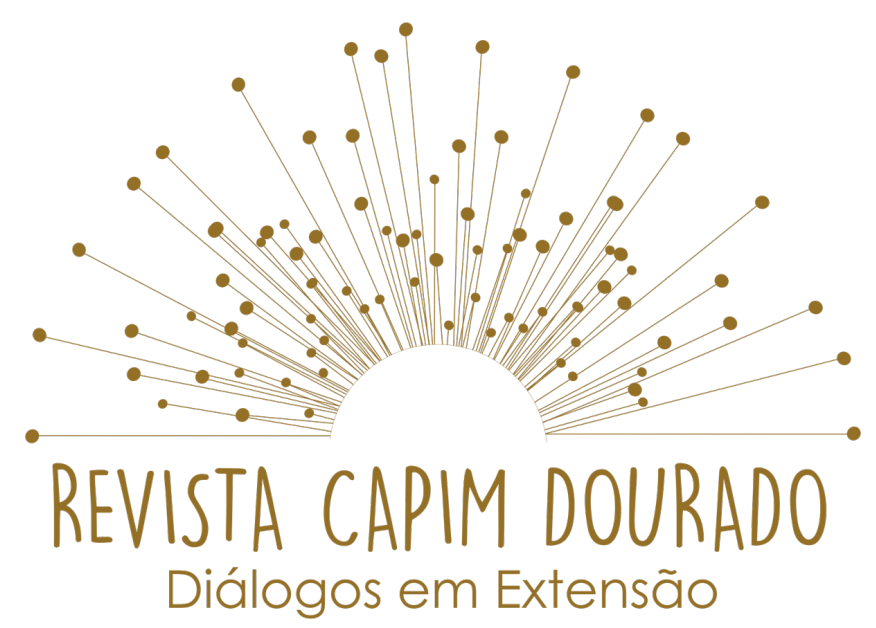

ISSN n² 2595-7341

Vol. 3, n. 1, Janeiro-Abril, 2020

DOI: http://dx.doi.org/10.20873/uft.2595-7341.2020v3n1p95

frequency (number of days per week and at the weekend) of using television separately, video games and computer (at leisure and for teenage activities) on weekdays and weekends, considering the week prior to data collection, physical activity was measured by a validated questionnaire Adolescents reported the frequency (days / week) duration (minutes / day) of physical activities practiced in the week prior to data collection. 69 students participated in the study with an average age of 15.4 years $(S D=0.2$ ), from the 6th and 9th grade of Elementary School and 1st and 3rd high school, of which 52.2\% were girls. The students performed an average of $256.5 \mathrm{~min} . /$ day $(S D=29.9)$ of physical activities remained $748.8 \mathrm{~min} . /$ day (SD $=86.5$ ) watching television and $553.6 \mathrm{~min} . /$ day (SD $=75.9$ ). The results indicate that the adolescents from Tocantinópolis / TO have little physical activity and high levels of sedentary behavior.

KEYWORDS: Physical Activity. Physical aptitude. Sedentary lifestyle.

\section{RESUMEN}

Los estudios han demostrado que el nivel de actividad física está relacionado con el mantenimiento de niveles saludables de aptitud física, y los niveles bajos de aptitud física se han asociado con el riesgo de desarrollar enfermedades cardiovasculares, mortalidad por todas las causas, tabaquismo y consumo de bebidas. alcohólico. Y puede describir la prevalencia de actividad física, conductas sedentarias y sus posibles asociaciones con los factores socioeconómicos y antropométricos de los escolares de la ciudad de Tocantinópolis (TO). La recogida incluyó la aplicación de un cuestionario en forma de entrevista presencial, una medida de los comportamientos sedentarios considerando la duración (horas por día) y la frecuencia (número de días por semana y fin de semana) de usar la televisión por separado., videojuegos y computadora (en el tiempo libre y para actividades de adolescentes) en días laborables y fines de semana, considerando la semana anterior a la recolección 


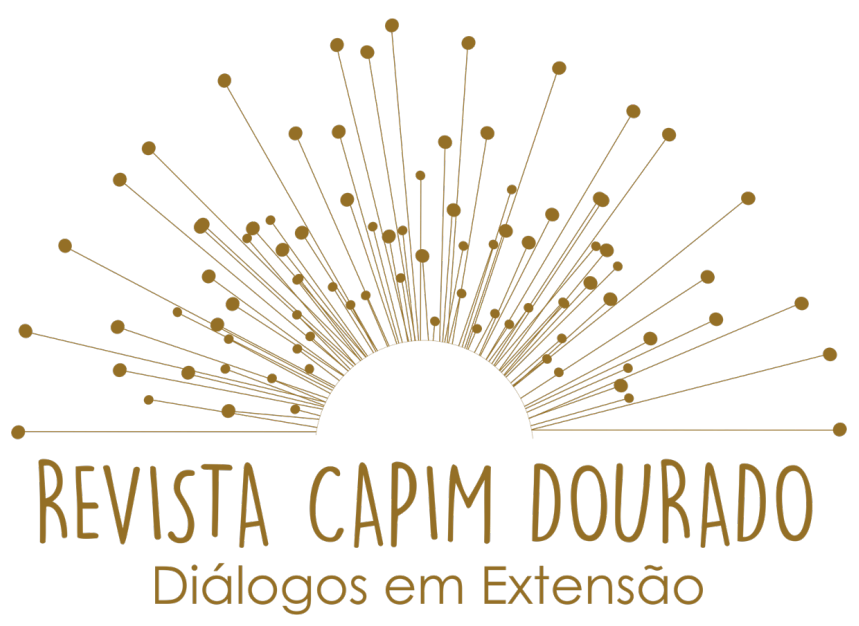

ISSN n² 2595-7341

Vol. 3, n. 1, Janeiro-Abril, 2020

DOI: http://dx.doi.org/10.20873/uft.2595-7341.2020v3n1p95

de datos, la actividad física se midió mediante un cuestionario validado Los adolescentes informaron la frecuencia (días / semana) duración (minutos / día) de actividades físicas practicadas en la semana anterior a la recolección de datos. Participaron del estudio 69 estudiantes con una edad promedio de 15,4 años (DE $=0,2$ ), de $6^{\circ}$ y $9^{\circ}$ de Primaria y $1^{\circ}$ y $3^{\circ}$ de Bachillerato, de los cuales el $52,2 \%$ eran niñas. Los estudiantes realizaron un promedio de $256,5 \mathrm{~min}$./día $(D E=29,9)$ de actividades físicas permaneciendo 748,8 min./día ( $D E=86,5)$ viendo televisión y 553,6 min./día ( $D E=75,9$ ). Los resultados indican que los adolescentes de Tocantinópolis / TO tienen poca actividad física y altos niveles de sedentarismo.

PALABRAS CLAVE: Actividad física. Aptitud física. Estilo de vida sedentario.

Recebido em: 01.10.2019. Aceito em: 09.10.2019. Publicado em: 01.01.2020. 


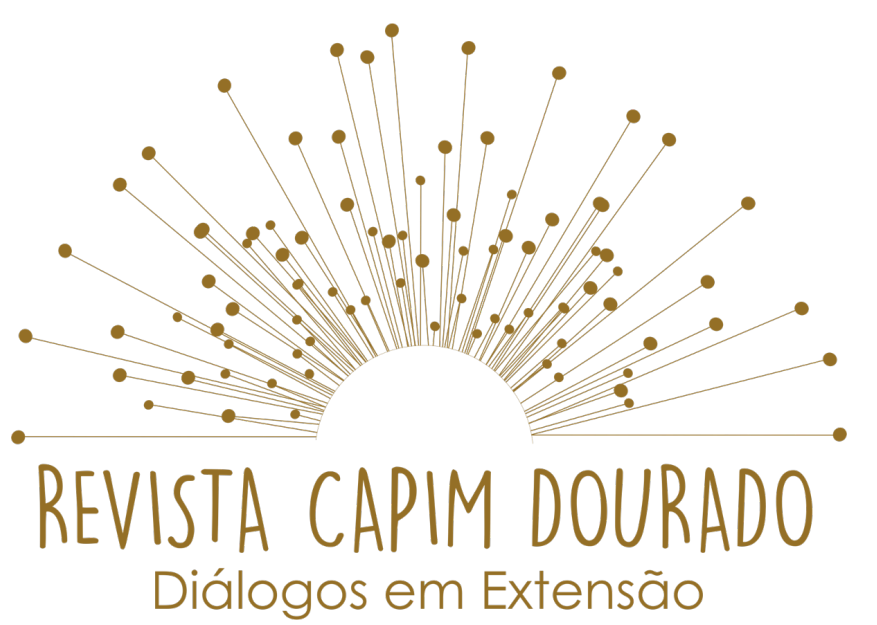

ISSN n² 2595-7341

Vol. 3, n. 1, Janeiro-Abril, 2020

DOI: http://dx.doi.org/10.20873/uft.2595-7341.2020v3n1p95

\section{INTRODUÇÃO}

Estudos têm demonstrado que o nível de atividade física está relacionado à manutenção de níveis saudáveis de aptidão física e, baixos níveis de aptidão física têm sido associados a o risco de desenvolver doenças cardiovasculares, de mortalidade por todas as causas (ERIKSSEN et al, 2001), ao tabagismo e ao uso de bebidas alcoólicas (PATE et al, 1996). Assim, é importante realizar estudos que analisem o tempo despendido em comportamentos sedentários, o nível de atividade física e a aptidão física de adolescentes em diversas faixas etárias e realizar estudos em outros países e regiões, dado que essas características mudam conforme a idade e a realidade social, econômica e cultural interfere nos hábitos de cada população, em cada país e região do Brasil. O objetivo deste estudo foi descrever a prevalência de atividade física, comportamentos sedentários e suas possíveis associações com os fatores socioeconômicos e antropométricos de escolares da cidade de Tocantinópolis (TO).

\section{METODOLOGIA}

Este projeto é um recorte do "PROJETO MAIS ATIVO: Acompanhamento Da Aptidão Física E Qualidade De Vida Relacionada À Saúde Em Escolares De Tocantinópolis (TO)". O Projeto Mais Ativo caracteriza-se como longitudinal, de base escolar, realizado entre 2018 e 2020. Tem como população de referência adolescentes do $6^{\circ}$ e $9^{\circ}$ ano do ensino fundamental II e $1^{\circ}, 2^{\circ}$ e $3^{\circ}$ ano do ensino médio, de ambos os sexos, regularmente matriculados em escolas municipais e estaduais do município de Tocantinópolis (TO). A coleta incluiu a aplicação de 


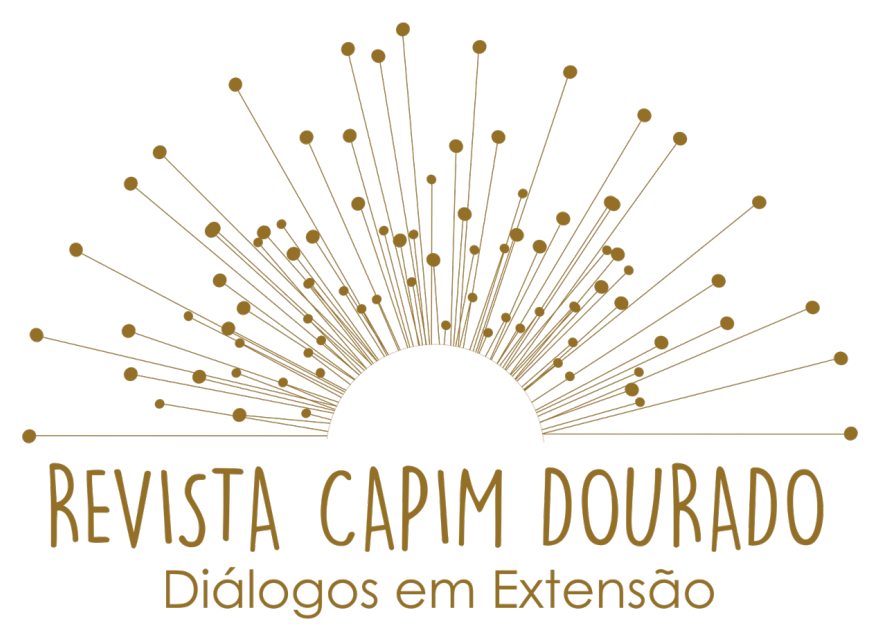

ISSN n² 2595-7341

Vol. 3, n. 1, Janeiro-Abril, 2020

DOI: http://dx.doi.org/10.20873/uft.2595-7341.2020v3n1p95

um questionário na forma de entrevista face a face. A medida dos comportamentos sedentários considerou a duração (horas por dia) e a frequência (quantidade de dias na semana e no fim de semana) do uso, separadamente, de televisão, videogames e computador (no lazer e para atividades adolescentes) em dias de semana e fim de semana, considerando a semana anterior à coleta de dados. A atividade física foi mensurada por questionário validado. Os adolescentes informaram a frequência (dias/semana) e a duração (minutos/dia) das atividades físicas praticadas na semana anterior à coleta de dados. Os dados foram digitados em duplicata no Microsoft Excel 97 - 2003. Para análise descritiva das variáveis numéricas, será utilizado o cálculo da média e desvio padrão. Para as variáveis mensuradas em escala nominal e ordinal, será utilizada a distribuição por frequências. Para todas as medidas, será adotado um intervalo de confiança de $95 \%$.

\section{FUNDAMENTAÇÃO TEÓRICA}

Os estudos de Tenório et al identificou que local de residência (rural/urbano), turno de aulas, status ocupacional e participação nas aulas de educação física foram fatores associados a nível insuficiente de atividade física. O estudo de Farias Júnior et al percebeu que os fatores diretamente associados à prática de atividade física foram maior escolaridade do pai para o sexo masculino, e da mãe, para o feminino; percepção positiva de saúde e participar das aulas de educação física. Já o estudo de Dias et al, demonstrou que residir 


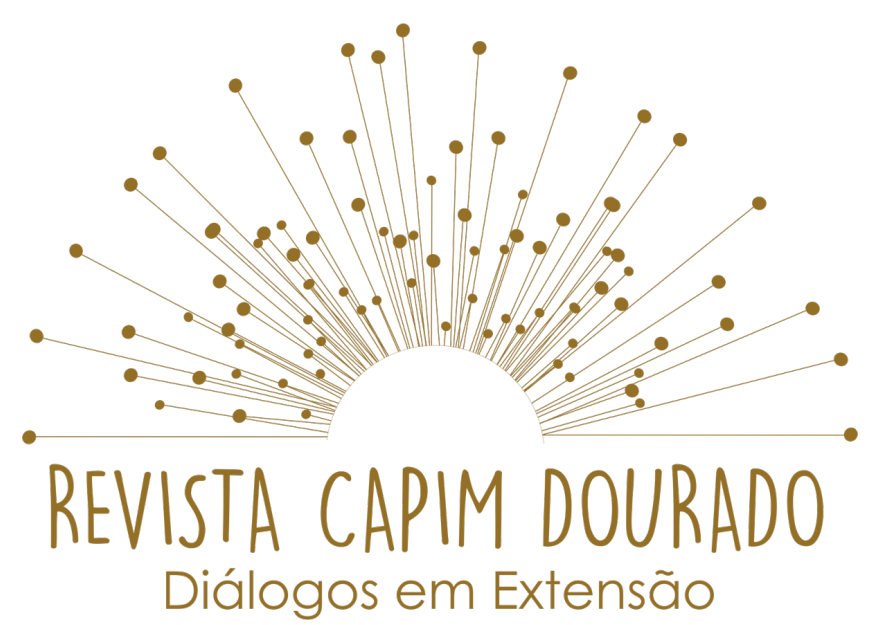

ISSN n² 2595-7341

Vol. 3, n. 1, Janeiro-Abril, 2020

DOI: http://dx.doi.org/10.20873/uft.2595-7341.2020v3n1p95

no interior foi um dos fatores associados ao comportamento sedentário. É interessante notar que esses estudos foram realizados em Pernambuco, Cuiabá e João Pessoa. Dessa forma, pode-se inferir que, das mudanças culturais entre as regiões brasileiras, como Nordeste e Centro-Oeste, derivam resultados comportamentais diferentes para determinados grupos de adolescentes. Assim, provavelmente futuras intervenções devem considerar essas diferenças ao elaborar um plano de ação que diminua o tempo em comportamento sedentário e aumente o nível de atividade física.

\section{RESULTADOS FINAIS}

Participaram do estudo 69 escolares com idade média de 15,4 anos (DP $=0,2)$, dos $6^{\circ}$ e $9^{\circ}$ ano do Ensino Fundamental e $1^{\circ}$ e $3^{\circ}$ ensino médio, dos quais $52,2 \%$ eram meninas. Em relação à escolaridade da mãe, 63,3\% eram analfabetas ou cursaram até $4^{\text {a }}$ série e apenas $36,8 \%$ estudaram até o ensino fundamental. Quanto aos pais, $55,9 \%$ eram analfabetos ou cursaram até $4^{\text {a }}$ série e $44,1 \%$ até o fundamental. Os escolares realizavam em média 256,5 min./dia ( $D P=29,9)$ de atividades físicas e permaneciam $748,8 \mathrm{~min}$./dia ( $D P=86,5)$ assistindo televisão e 553,6 min./dia ( $D P=75,9)$ em comportamentos sedentários no geral. Estes dados correspondem a um dos primeiros levantamentos sobre estilo de vida de adolescentes da região do Bico do Papagaio, região caracteristicamente de menor nível socioeconômico, que apresenta dificuldades para rede de transporte público e diferenças culturais, devido à proximidade do limite territorial com o 


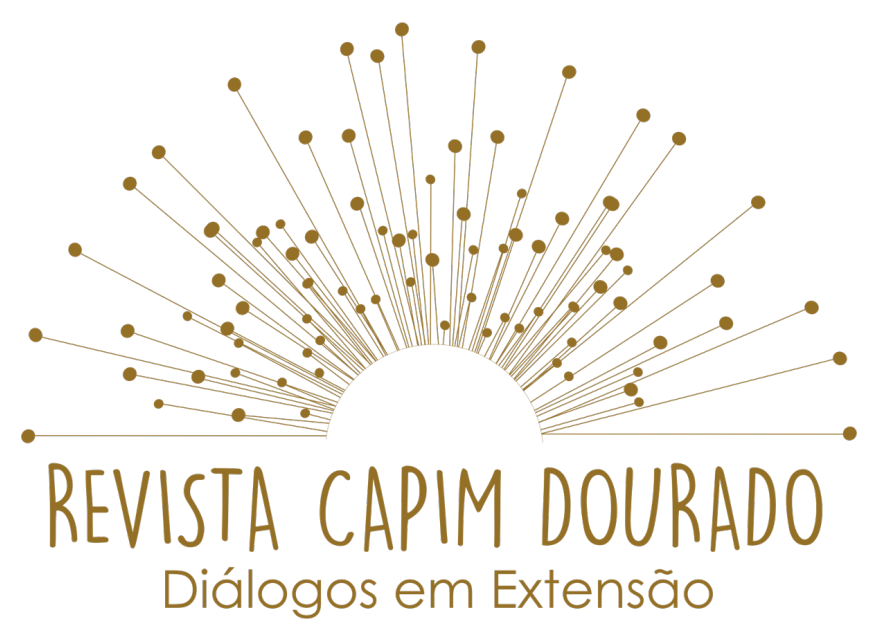

ISSN n² 2595-7341

Vol. 3, n. 1, Janeiro-Abril, 2020

DOI: http://dx.doi.org/10.20873/uft.2595-7341.2020v3n1p95

Pará e o Maranhão. Nesse sentido, seria possível esperar diferentes comportamentos e, talvez, um estilo de vida mais fisicamente ativo.

\section{CONSIDERAÇÕES FINAIS}

Os resultados indicam que os adolescentes de Tocantinópolis/TO apresentam pouco tempo de atividade física e altos índices de comportamentos sedentários.

\section{REFERÊNCIAS}

Erikssen G. Physical fitness and changes in mortality. Sports medicine. 2001;31(8):571-6.

Pate RR, Heath GW, Dowda M, Trost SG. Associations between physical activity and other health behaviors in a representative sample of US adolescents. American journal of public health. 1996;86(11):1577-81.

Tenório M, Barros Md, Tassitano R, Bezerra J, Tenório J, Hallal P. Atividade física e comportamento sedentário em adolescentes estudantes do ensino médio. Rev Bras Epidemiol. 2010;13(1):105-17.

Farias Júnior JCd, Lopes AdS, Mota J, Hallal PC. Prática de atividade física e fatores associados em adolescentes no Nordeste do Brasil. Revista de Saúde Pública. 2012;46:505-15.

Dias PJP, Domingos IP, Ferreira MG, Muraro AP, Sichieri R, Gonçalves-Silva RMV. Prevalência e fatores associados aos comportamentos sedentários em adolescentes. Revista de Saúde Pública. 2014;48:266-74. 